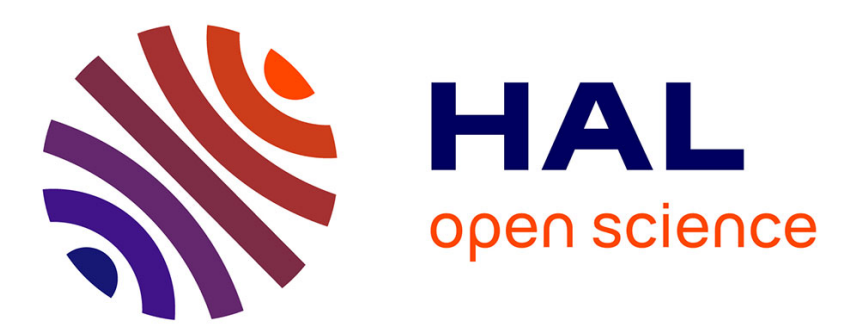

\title{
Spectres de pertes d'énergie caractéristiques sur Ta et Pt sous hydrogène
}

C. Le Gressus, D. Massignon, F. Pellerin

\section{To cite this version:}

C. Le Gressus, D. Massignon, F. Pellerin. Spectres de pertes d'énergie caractéristiques sur Ta et Pt sous hydrogène. Journal de Physique Lettres, 1979, 40 (13), pp.309-312. 10.1051/jphyslet:019790040013030900 . jpa-00231631

\section{HAL Id: jpa-00231631 https://hal.science/jpa-00231631}

Submitted on 1 Jan 1979

HAL is a multi-disciplinary open access archive for the deposit and dissemination of scientific research documents, whether they are published or not. The documents may come from teaching and research institutions in France or abroad, or from public or private research centers.
L'archive ouverte pluridisciplinaire HAL, est destinée au dépôt et à la diffusion de documents scientifiques de niveau recherche, publiés ou non, émanant des établissements d'enseignement et de recherche français ou étrangers, des laboratoires publics ou privés. 


\title{
Spectres de pertes d'énergie caractéristiques sur Ta et Pt sous hydrogène
}

\author{
C. Le Gressus, D. Massignon et F. Pellerin \\ Division de Chimie, Service de Chimie-Physique, CEN-Saclay, BP No 2, 91190 Gif sur Yvette, France
}

(Reçu le 5 février 1979, révisé le 25 avril 1979, accepté le 8 mai 1979)

\begin{abstract}
Résumé. - L'hydrogène adsorbé à la température ambiante sur le tantale et sur le platine a été analysé par spectrométrie de pertes d'énergie caractéristiques. Sur les deux substrats, la présence d'hydrogène est responsable d'une perte à $13 \mathrm{eV}$ constante en énergie sur les différents échantillons et d'intensité variable avec la pression du gaz. Cette perte d'énergie caractéristique est attribuée à l'excitation des transitions entre niveaux électroniques

$$
{ }^{1} \Sigma \mathrm{g}^{+} \rightarrow{ }^{1} \Sigma \mathrm{u}^{+} \text {et }{ }^{1} \Sigma \mathrm{g}^{+} \rightarrow{ }^{1} \Pi \mathrm{u}
$$

de la liaison moléculaire de l'hydrogène plutôt qu'à l'excitation de la liaison métal-hydrogène.

Abstract. - Hydrogen adsorbed at room temperature on tantalum and platinum samples has been analysed by the mean of Energy Loss Spectroscopy (ELS). On both samples, hydrogen adsorption is correlated with an ELS peak located at $13 \mathrm{eV}$ below the elastic peak. This energy loss is attributed to the excitation of ${ }^{1} \Sigma \mathrm{g}^{+} \rightarrow{ }^{1} \Sigma \mathbf{u}^{+}$and ${ }^{1} \mathrm{Sg}^{+} \rightarrow{ }^{1} \Pi \mathrm{u}$ transitions of molecular hydrogen.
\end{abstract}

1. Introduction. - Le spectre des pertes d'énergie (ELS) enregistré en réflexion sur un échantillon contaminé, soit par une impureté à l'état solide $(\mathrm{C}, \mathrm{S}, \mathrm{Cl} \ldots)$, soit par un gaz adsorbé en surface, présente un certain nombre de pics caractéristiques de ces éléments contaminants. Ces pics sont situés à des énergies indépendantes de la nature du substrat.

Des études antérieures de pertes d'énergie sur le graphite, sur une couche organique ou sur du carbone ségrégé sur l'aluminium [1-5], ont montré, en bon accord avec les études optiques, que certaines de ces pertes peuvent être attribuées aux transitions

$$
\Pi \rightarrow \Pi^{*}(6 \mathrm{eV}) \text { et } \sigma \rightarrow \sigma^{*}(20-25 \mathrm{eV})
$$

des orbitales moléculaires de la liaison carbonecarbone.

D'autre part, lors de l'adsorption de CO sur les métaux de transition, une perte d'énergie caractéristique à $14 \mathrm{eV}$ a été signalée [6]. Elle est habituellement interprétée comme une excitation de la liaison moléculaire de $\mathrm{CO}\left(5 \sigma \rightarrow 2 \Pi^{*}\right)$.

Nous nous proposons de montrer que l'hydrogène adsorbé sur le tantale ou sur le platine est responsable de l'apparition d'une perte d'énergie caractéristique située à $13 \mathrm{eV}$ en dessous du pic élastique et que cette perte est due à l'excitation de la liaison moléculaire de l'hydrogène.
2. Expérimentation. - L'appareillage utilisé est composé d'un analyseur cylindrique à miroir (CMA) dont on a déterminé la bande passante en fonction de l'énergie de l'électron analysé [4]

$$
(\Delta E=k E, k \simeq 0,3 \%),
$$

et d'un faisceau électronique coaxial dont on a mesuré la dispersion énergétique (largeur à mi-hauteur $\simeq 750 \mathrm{meV}$ ).

La distribution spectrale des énergies est analysée selon une méthode de détection utilisant un amplificateur Lock-in, associé soit à une modulation de la brillance du faisceau primaire, soit à une modulation de la tension appliquée sur l'analyseur [7]. On peut ainsi enregistrer $E n(E) \quad E \frac{\mathrm{d} n(E)}{\mathrm{d} E}$ en fonction de $E$.

Le courant primaire $I_{\mathrm{p}}$ est mesuré dans une cage de Faraday avant l'enregistrement du spectre. Il a été maintenu constant pour tous les spectres présentés $\left(I_{\mathrm{p}}=5 \times 10^{-8} \mathrm{~A}\right)$. Dans les conditions de tension d'accélération primaire $E_{\mathrm{p}}$ utilisées ici

$$
\left(100 \leqslant E_{\mathrm{p}} \leqslant 200 \mathrm{eV}\right),
$$

le diamètre $\varnothing \mathrm{du}$ spot électronique primaire est de l'ordre de $0,1 \mathrm{~mm}$ ou plus. La densité de courant primaire reste donc toujours inférieure à $5 \times 10^{-4} \mathrm{~A} . \mathrm{cm}^{-2}$. Les spectres étant enregistrés en $100 \mathrm{~s}$, la dose reçue 
par l'échantillon pendant l'enregistrement d'un spectre $\left(5 \times 10^{17} \mathrm{e}^{-} . \mathrm{cm}^{-2}\right)$ est très faible et les modifications du substrat (échauffement, champ électrique local) ou de l'adsorbat (désorption induite par le faisceau primaire) sont négligeables. La section efficace de désorption stimulée est en moyenne de $10^{-20} \mathrm{~cm}^{2}$ à $100 \mathrm{eV}$ [11], valeur très inférieure aux sections efficaces d'interaction électron-molécule $\left(\sim 10^{-16} \mathrm{~cm}^{2}\right)$, et aux sections efficaces d'ionisation en couche profonde ( 10 $\left.0^{-19} \mathrm{~cm}^{2}\right)$.

Les échantillons polycristallins de Ta et $\mathrm{Pt}(99,99 \%)$ sont introduits sous ultra-vide, chauffés à $600^{\circ} \mathrm{C}$ et soumis à une abrasion ionique superficielle $\left(\mathrm{Ar}^{+}\right.$, $3 \mathrm{keV}, 10^{-5} \mathrm{~A} / \mathrm{cm}^{2}$ ). Le retour de l'échantillon à la température ambiante est de 1 heure environ; il a donc été nécessaire de continuer l'abrasion ionique pendant le refroidissement de celui-ci pour éviter une contamination possible. Après ce traitement, la contamination résiduelle par l'oxygène et le carbone n'est plus détectable en spectrométrie Auger. Elle peut donc être estimée inférieure au millième de monocouche lorsque le spectre est enregistré dans le mode $\operatorname{En}(E)$.

L'atmosphère dans le bâti est contrôlée en permanence par spectrométrie de masse. La pression partielle de $\mathrm{CO}$ reste inférieure ou égale à $10^{-10}$ torr pendant toute la durée de l'expérience.

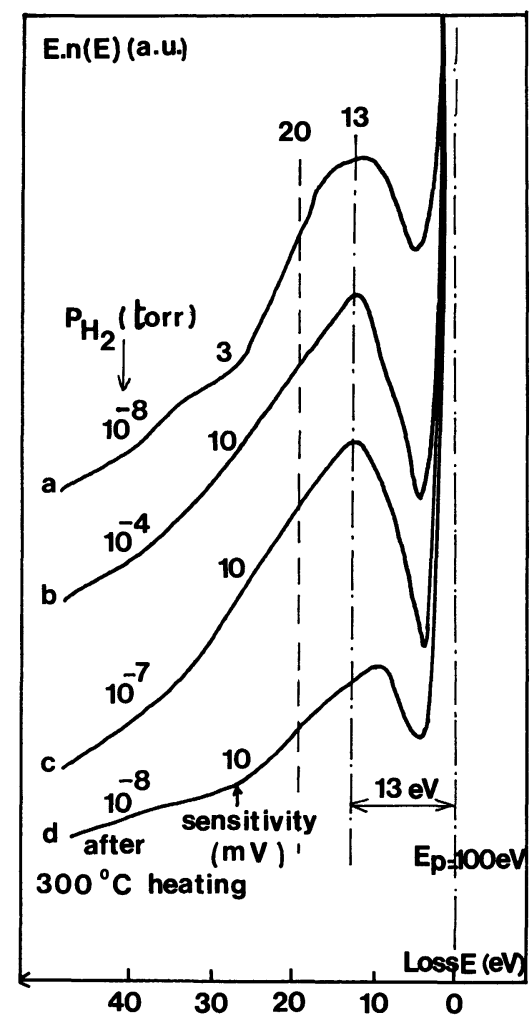

Fig. 1. - Spectres de pertes d'énergie caractéristiques sur le tantale dans le mode $E n(E)\left(E_{\mathrm{p}}=100 \mathrm{eV}, I_{\mathrm{p}}=5 \times 10^{-8} \mathrm{~A}\right)$. (a) Ta propre $p \mathrm{H}_{2}=10^{-8}$ torr ; (b) $p \mathrm{H}_{2}=10^{-4}$ torr ; (c) $p \mathrm{H}_{2}=10^{-7}$ torr après pompage; $(d) p \mathrm{H}_{2}=10^{-8}$ torr après chauffage.

$\left[E n(E)\right.$ spectrum ; (a) clean Ta $p \mathrm{H}_{2}=10^{-8}$ torr ; (b) $p \mathrm{H}_{2}=10^{-4}$ torr ; (c) $p \mathrm{H}_{2}=10^{-7}$ torr after pumping down; (d) $10^{-8}$ torr after heating.]
3. Résultats. - On enregistre les spectres de pertes d'énergie caractéristiques sur $\mathrm{Ta}$ pur dans le mode $E n(E)$ (Fig. $1 a, E_{\mathrm{p}}=100 \mathrm{eV}, I_{\mathrm{p}}=5 \times 10^{-8} \mathrm{~A}$ ), et dans le mode $E \mathrm{~d} n(E) / \mathrm{d} E$ (Fig. $2 a, E_{\mathrm{p}}=100 \mathrm{eV}$, $I_{\mathrm{p}}=5 \times 10^{-8} \mathrm{~A}$; modulation $\left.1 \mathrm{Vptp}\right)$, et sur Pt dans le mode $E n(E)$ (Fig. $3 a, E_{\mathrm{p}}=200 \mathrm{eV}, I_{\mathrm{p}}=5 \times 10^{-8} \mathrm{~A}$ ).

On enregistre ensuite les spectres de pertes d'énergie caractéristiques pour diverses pressions d'hydrogène dans le bâti (jusqu'à $7 \times 10^{-4}$ torr pour Pt et $10^{-4}$ torr pour Ta).

Dans le cas du platine (Fig. $3 b, c, d$, mêmes conditions expérimentales que $3 a$ ), une perte d'énergie apparaît à $13 \mathrm{eV}$ du pic élastique. L'intensité de cette perte d'énergie croît avec la pression d'hydrogène dans le bâti. La valeur observée de cette intensité décroît de façon réversible avec la pression d'hydrogène.

Dans le cas du tantale, une perte d'énergie apparaît également à $13 \mathrm{eV}$ du pic élastique (Figs. $1 b, 2 b$, mêmes conditions que $1 a, 2 a$ ). Les pics Auger de valence du tantale (Fig. $2 b$ ) disparaissent. Lorsqu'on repompe (Figs. 1c, 2c), la perte d'énergie à $13 \mathrm{eV}$

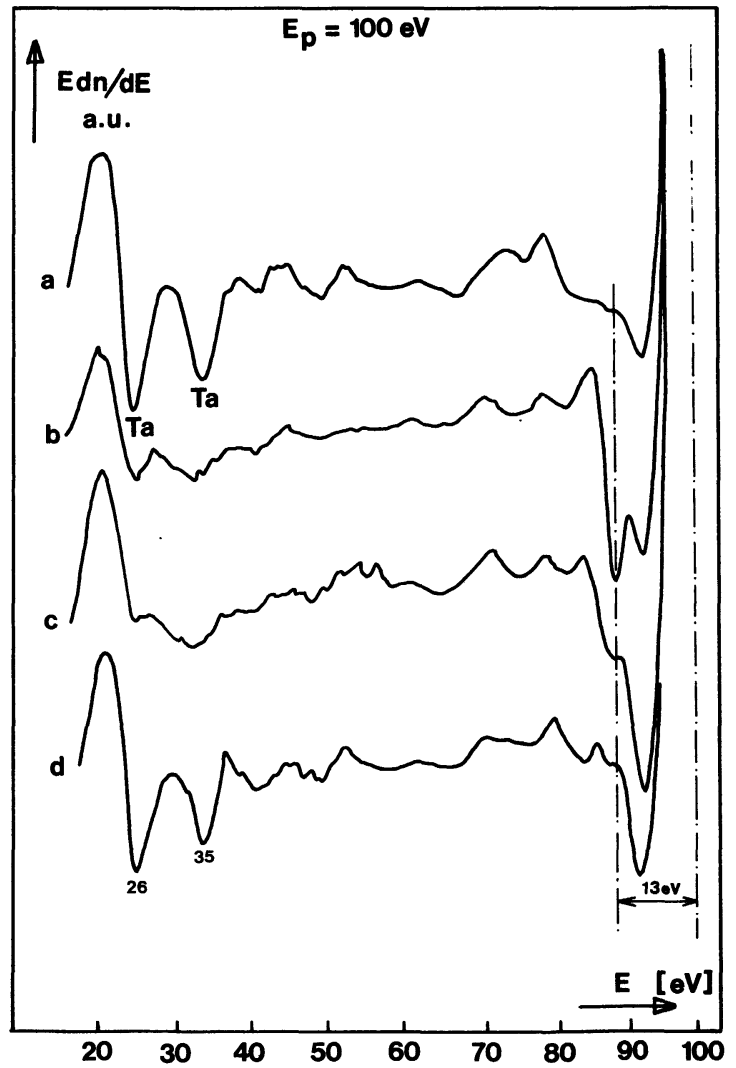

Fig. 2. - Spectres de pertes d'énergie caractéristiques sur le tantale dans le mode $E \frac{\mathrm{d} n(E)}{\mathrm{d} E}\left(E_{\mathrm{p}}=100 \mathrm{eV}, I_{\mathrm{p}}=5 \times 10^{-8} \mathrm{~A}\right.$, mod. IV). (a) Ta propre $p \mathrm{H}_{2}=10^{-8}$ torr; (b) $p \mathrm{H}_{2}=10^{-4}$ torr; (c) $p \mathrm{H}_{2}=10^{-7}$ torr après pompage; (d) $p \mathrm{H}_{2}=10^{-8}$ torr après chauffage.

$\left[E \frac{\mathrm{d} n(E)}{\mathrm{d} E}\right.$ spectrum; (a) clean $\mathrm{Ta} ;(b) p \mathrm{H}_{2}=10^{-4}$ torr ; (c) $p \mathrm{H}_{2}=10^{-7}$ torr after pumping down ; (d) $10^{-8}$ torr after heating.] 


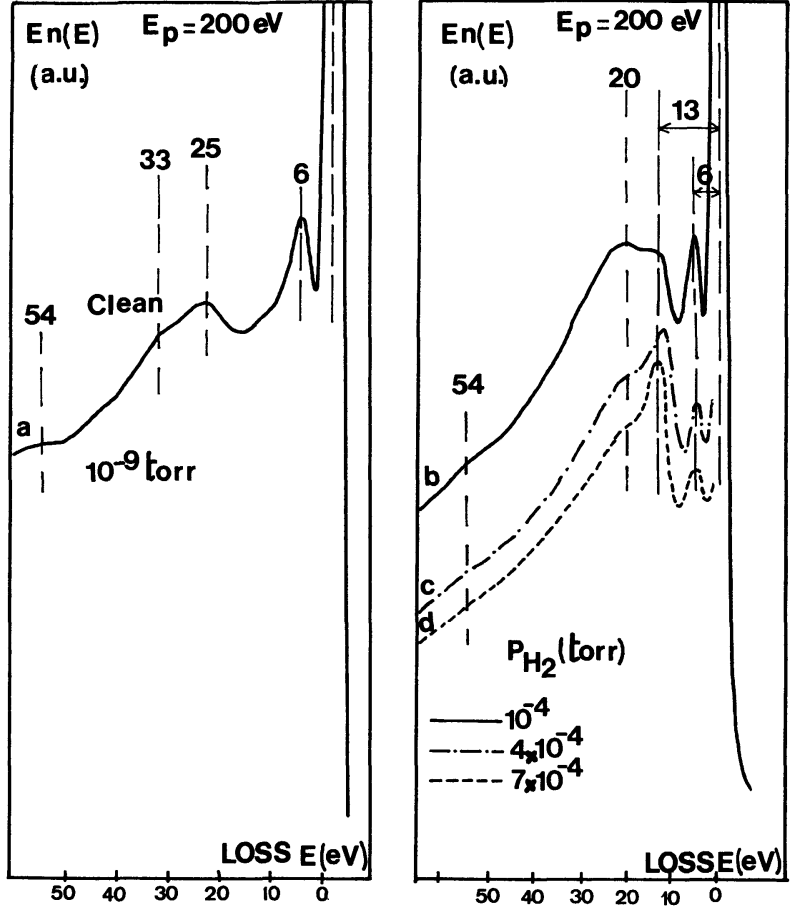

Fig. 3. - Spectres de pertes d'énergie caractéristiques sur le platine dans le mode $E n(E)\left(E_{\mathrm{p}}=200 \mathrm{eV}, I_{\mathrm{p}}=5 \times 10^{-8} \mathrm{~A}\right)$. (a) Pt propre $p=10^{-9}$ torr; (b) $p \mathrm{H}_{2}=10^{-4}$ torr ;

(c) $p \mathrm{H}_{2}=4 \times 10^{-4}$ torr; (d) $p \mathrm{H}_{2}=7 \times 10^{-4}$ torr.

$\left[E n(E)\right.$ spectrum; (a) clean $\mathrm{Pt}$; (b) $p \mathrm{H}_{2}=10^{-4}$ torr ; (c) $p \mathrm{H}_{2}=$ $4 \times 10^{-4}$ torr ; (d) $p \mathrm{H}_{2}=7 \times 10^{-4}$ torr. $]$

diminue mais les pics Auger de valence du tantale ne réapparaissent pas. Une analyse SIMS montre alors un pic $\mathrm{H}^{+}$intense. Il faut chauffer l'échantillon (Figs. $1 d, 2 d, 300^{\circ} \mathrm{C}, 5 \mathrm{~min}$ ) pour que la perte à $13 \mathrm{eV}$ disparaisse elle aussi et pour que les pics Auger réapparaissent. L'analyse SIMS ne révèle plus alors de pic $\mathrm{H}^{+}$. Les spectres de pertes d'énergie caractéristiques ainsi obtenus sont différents des spectres de départ (Figs. 1a, 2a). Il est probable que pour les spectres $1 a$ et $2 a$, la quantité d'hydrogène adsorbé en surface n'est pas négligeable. Le vide résiduel de $10^{-8}$ torr est en effet composé essentiellement d'hydrogène et le temps de séjour dans ce vide est assez important $(10 \mathrm{~min})$.

Dans les deux cas, au cours de l'adsorption d'hydrogène une perte peu intense apparaît à $20 \mathrm{eV}$, elle est irréversible et correspond à une faible contamination durable de la surface par du carbone [4].

4. Discussion. - Une perte d'énergie caractéristique située $15 \mathrm{eV}$ environ en dessous du pic élastique a été observée par Küppers [8] sur Ni (110) et par Christmann et al. sur Pt (111) [9] lors de l'adsorption d'hydrogène. L'énergie de cette perte n'est pas tout à fait la même pour ces deux substrats. Elle est attribuée par ces auteurs à l'excitation de la liaison métalhydrogène. La perte d'énergie observée l'a été sur une surface saturée en hydrogène chimisorbé. Cette saturation se produit à la température ambiante pour 40 min à $3 \times 10^{-8}$ torr $\mathrm{H}_{2}$ sur $\mathrm{Ni}(110)$ [8].

La perte d'énergie caractéristique que nous observons à $13 \mathrm{eV}$ environ semble avoir une origine physique différente de celle rapportée par les auteurs précédents pour les raisons suivantes :

(i) La valeur de la perte d'énergie caractéristique observée ici est indépendante du substrat. Toutefois, la résolution de l'analyseur à ces énergies $(\sim 0,5 \mathrm{eV})$ et le fait que $\mathrm{Ta}$ et $\mathrm{Pt}$, tous les deux métaux de transitions, ont des structures électroniques proches, pourrait laisser penser que la valeur de la perte d'énergie ne varie pas assez pour être détectée. Cependant, une étude en cours sur l'aluminium, et qui sera publiée prochainement, montre que dans ce cas également, la perte d'énergie lors de l'adsorption d'hydrogène apparaît à $13 \mathrm{eV}$ pour des pressions d'hydrogène équivalentes. La structure électronique de l'aluminium étant très différente de celle de $\mathrm{Ta}$ et $\mathrm{Pt}$, la perte d'énergie caractéristique à $13 \mathrm{eV}$ semble bien être indépendante du substrat.

(ii) L'intensité de la perte d'énergie caractéristique à $13 \mathrm{eV}$ croît avec la pression d'hydrogène jusqu'à la limite supérieure de pression que peut supporter notre installation $\left(7 \times 10^{-4}\right.$ torr) (Fig. $\left.3 d\right)$. Il ne semble pas y avoir, dans le cas de cette perte d'énergie, de phénomène de saturation, contrairement aux expériences de Küppers et Christmann. D'autre part, la pression d'hydrogène atteinte dans notre bâti est environ $10^{4}$ fois supérieure à celle de ces auteurs $\left(3 \times 10^{-8}\right.$ torr $\left.\mathrm{H}_{2}\right)$. Nos conditions expérimentales sont donc très différentes des leurs.

(iii) La valeur de l'intensité de la perte d'énergie à $13 \mathrm{eV}$ est réversible avec la pression d'hydrogène. Elle est totalement réversible dans le cas du platine. Elle est en partie réversible seulement dans le cas du tantale. Ceci semble indiquer une très faible énergie de liaison de l'hydrogène responsable de cette perte d'énergie et exclut aussi que ce pic soit attribué à $\mathrm{CO}$, dont l'énergie de liaison est très élevée [6] et la perte irréversible.

L'ensemble de ces conditions expérimentales conduit à attribuer la perte d'énergie à $13 \mathrm{eV}$ à l'excitation de la liaison moléculaire de l'hydrogène plutôt qu'aux liaisons métal-hydrogène. Les transitions

$$
{ }^{1} \Sigma \mathrm{g}^{+} \rightarrow{ }^{1} \Sigma \mathrm{u}^{+} \text {et }{ }^{1} \Sigma \mathrm{g}^{+} \rightarrow{ }^{1} \Pi \mathrm{u}
$$

ont des valeurs théoriques correspondant bien aux valeurs observées $(11,4$ et $12,4 \mathrm{eV})$ et des sections efficaces d'excitation à $100 \mathrm{eV}$ importantes $\left(10^{-17} \mathrm{~cm}^{2}\right)$ [10], ce qui permet de rendre compte de la forte intensité de cette perte par rapport à celle observée par Küppers et Christmann.

L'adsorption d'hydrogène sur les métaux à la température ambiante comporte probablement deux étapes :

- une première étape de chimisorption de l'hydrogène (sous forme atomique). Elle a lieu pour des quantités d'hydrogène adsorbé faibles. Cette étape explique les résultats de Küppers et Christmann. 
La perte d'énergie à $15 \mathrm{eV}$ qu'ils observent serait due à l'excitation de la liaison métal-hydrogène. Ceci expliquerait la saturation de l'intensité de la perte pour des quantités d'hydrogène adsorbé très inférieures aux nôtres (40 min à $3 \times 10^{-8}$ torr [8]) et sa non-réversibilité.

- La deuxième étape aurait lieu pour des quantités d'hydrogène adsorbé beaucoup plus fortes. L'hydrogène moléculaire pourrait se lier faiblement à l'hydrogène chimisorbé au cours de la première étape. Cet hydrogène moléculaire ne verrait donc pas directement l'échantillon. Ceci expliquerait la valeur constante de la perte d'énergie à $13 \mathrm{eV}$ quel que soit le substrat, la non-saturation de la perte pour des quantités d'hydrogène adsorbé très fortes et la réversibilité de celle-ci. L'interaction entre l'hydrogène chimisorbé et l'hydrogène moléculaire serait probablement du type dipôle-dipôle (liaison de Van der Waals).

$\mathrm{Ce}$ modèle semble très bien adapté aux résultats obtenus sur le platine. Dans le cas du tantale toutefois, l'interaction tantale-hydrogène est beaucoup plus forte. Il faut en effet chauffer l'échantillon pour faire disparaître totalement la perte d'énergie à $13 \mathrm{eV}$. Ce couplage plus fort entre le tantale et l'hydrogène se traduit, non par un déplacement de l'énergie de la perte, mais par un élargissement très important de celle-ci. La largeur à mi-hauteur de la perte est de $5 \mathrm{eV}$ environ sur le platine (Fig. $3 d$ ) et de $15 \mathrm{eV}$ environ sur le tantale (Fig. 1b). Ceci confirme l'hypothèse du couplage molécule d'hydrogène-tantale plutôt que celle de la liaison atome d'hydrogène-tantale.

5. Conclusion. - Lorsqu'on analyse le spectre de pertes d'énergie caractéristiques de Ta et $\mathrm{Pt}$ sous pression d'hydrogène, il apparaît une perte d'énergie à $13 \mathrm{eV}$ en dessous du pic élastique. Cette perte d'énergie a une valeur en énergie constante quel que soit le substrat, elle croît avec la pression d'hydrogène sans phénomène de saturation et elle est réversible avec la pression d'hydrogène. Elle est attribuée aux transitions ${ }^{1} \Sigma \mathrm{g}^{+} \rightarrow{ }^{1} \Sigma \mathrm{u}^{+}$et ${ }^{1} \Sigma \mathrm{g}^{+} \rightarrow{ }^{1} \Pi \mathrm{u}$ de la molécule d'hydrogène. Ce travail pourrait permettre de distinguer l'hydrogène adsorbé sous forme moléculaire de l'hydrogène adsorbé sous forme atomique dans les études d'adsorption de l'hydrogène sur les métaux.

Remerciements. - Nous remercions M. le Pr. Levenson, Université de Missouri-Rolla, pour son soutien et ses conseils et M. Morin, Chef du Service de Chimie, CEA-DAM, chez qui ces expériences ont été faites.

\section{Bibliographie}

[1] SEVIER, K. D., Low energy electron spectroscopy, Ch. 8, p. 278.

[2] LANG, B., Surf. Sci. 66 (1977) 527.

[3] Gramari, D., Cazaux, S., Phys. C. 9 (1976) 421.

[4] Pellerin, F., Le Gressus, C., Surf. Sci. (1979), accepté pour publication.

[5] Isaacson, M., J. Chem. Phys. 56 (1972) 1803.

[6] NetZer, F. D., Matthew, J. A. D., Surf. Sci. 81 (1979) L 651.
[7] Le Gressus, C., Massignon, D., Sopizet, R., Surf. Sci. 68 (1977) 338.

[8] KüPPERS, J., Surf. Sci. 36 (1973) 53.

[9] Christmann, K., Ertl, G., Pignet, T., Surf. Sci. 54 (1976) 365.

[10] Handbuch der Physik, Vol. 37 (molecule I), (Springer) 1959, p. 339.

[11] MenZel, Surf. Sci. 47 (1975) 370. 